BULLETIN OF THE

AMERICAN MATHEMATICAL SOCIETY

Volume 77, Number 1, January 1971

\title{
GORDON T. WHYBURN
}

1904-1969

BY E. E. FLOYD AND F. B. JONES

Gordon Thomas Whyburn was born at Lewisville, Texas on January 7, 1904, the son of Thomas and Eugenia Elizabeth Whyburn. After attending the public schools of Lewisville, he went to the University of Texas, where he obtained the A.B. (1925), M.A. (1926) and Ph.D. (1927). In 1925, he married Lucille Smith, also from Lewisville and a mathematics student at Texas. His elder brother, William Marvin, was during the same years a mathematics student at Texas and on the way to his career in mathematics and administration. During the years 1927-1929, Whyburn was Adjunct Professor of Mathematics at Texas. He held a Guggenheim Fellowship in 19291930 and spent the year in Vienna, with trips to such European centers as Warsaw. Upon his return to the USA, he became Associate in Mathematics at The Johns Hopkins University. In 1934, Whyburn accepted the chairmanship of the Department of Mathematics at the University of Virginia and became Professor of Mathematics there. He lived in Charlottesville for the rest of his life, except for frequent summers teaching at Stanford, University of California, UCLA and the University of Colorado, and the year 1952-1953 on leave at Stanford and 1956-1957 on leave in England and Switzerland. The Whyburn's only child, Kenneth Gordon, now on the faculty in mathematics at the University of Washington, was born in 1944. Whyburn held the chairmanship until 1966, when he became the first member of the new Center for Advanced Studies in the Sciences at Virginia as well as retaining his position as Alumni Professor of Mathematics. He suffered a heart attack in 1966, but recovered and resumed a full schedule of teaching and research until his death of a sudden heart attack on September 8, 1969.

Whyburn was a very private man. He was quiet and shy, and remarkably gentle with students and family. But in moments of administrative crisis, he could be extremely tough when he had to be. A man of brilliance, with a remarkable speed in research, he nevertheless believed deeply in continuity and patience, and that it was the total record of accomplishment of a lifetime that mattered most.

I. His CAREer AS A LEADER

Whyburn was a brilliant scholar from the first. As a young chem- 
istry student he took calculus from R. L. Moore and continued to take Moore's courses and began to do research for Moore in the succeeding years. Moore kept steady pressure on him to switch to mathematics. After obtaining his Master's degree in chemistry, he did switch and obtained his Ph.D. in mathematics a year later. By that time he had already done a remarkable amount of research.

A year as a Guggenheim Fellow in Europe, when Whyburn was 25, was of particular importance to the development of his outlook on the mathematics world. He formed close ties with Kuratowski, Sierpiński and Hahn, and came to know Vietoris, Stoilow and numerous other European topologists. These contacts, together with the view of the young and ambitious American mathematical world of that time, helped give him a broad picture of the mathematical community.

In 1933, the University of Virginia began to interest Whyburn in a post as Chairman and Professor. The university was quite small and proud of its tradition as Jefferson's university. Its three professors of mathematics were retiring shortly. The university sought to use the opportunity to establish a first-rate research and graduate department. There were very few Ph.D. programs in the South, most of them were of token character, and only the programs at Texas and Rice were in any sense distinguished. Whyburn became excited about the prospects. For one thing, there were a few first-rate traditions in mathematics at Virginia-Sylvester had been a young faculty member there (although not a very happy one), the Annals of Mathematics had been founded there. Moreover he was excited by the prospect of locating first rate graduate departments more widely about the country, and came to see Virginia as a place for him to do his part.

The situation at Virginia inspired from Whyburn an ideal plan to fit the circumstances. One would get together a few young and congenial mathematicians of topflight accomplishments. Their fields should be differing but overlapping so that there would be beneficial contacts between them and so that the students would not be confronted with choosing between absolutely unrelated areas. The plan worked beautifully. E. J. McShane joined the Department in 1935, and G. A. Hedlund in 1939. The three of them ran a program of charm and high standards. The faculty never numbered more than six, the library was minimal, the facilities were very imperfect. But these young, vigorous men of high accomplishments ran an extremely effective program nevertheless.

In the years after World War II, the changes in American academia began to make their mark. Enrollments climbed, staffs grew, 
research specialization increased. Whyburn seemed to feel deeply that if the department was not to be transient then steady and continuous leadership was required from him. Until 1966 he continued to serve as chairman. For all but a few years he had no assistant chairman, but simply did many of the necessary details himself. For establishing and nurturing a research department, Whyburn ranks very high in contributions to Southern mathematics.

However, Whyburn's goal was not at all to serve Southern mathematics but to serve American mathematics as a whole. He must have come to know reasonably early his talent for organizationcomplete sympathy for first-rate research, a calm and dispassionate point of view, extreme quickness in getting to the core of an argument, willingness to handle dirty details. As the years passed, he contributed more and more of his talents to national organizations, most of all to the Society.

We will begin here with one of his early big committee assignments, in 1938-1939 as a member of the committee to make recommendations to the Society on an abstracting journal. With German periodicals perilously near being subject to Nazi orders, feelings were high that an American review journal was needed. On more permanent grounds as well, the argument was won and Mathematical Reviews came into being.

As the war came on, Whyburn became very concerned that a generation of young mathematicians not be wiped out, as a generation of English mathematicians had been in World War I. He also became concerned that American universities be kept alive and, in some minimal sense, functioning. In working to keep the University of Virginia open, he served as Director of the Premeteorological Training Program conducted at the university for the Army Air Forces. Nationally he became a member of the War Policy Committee, a joint committee of the AMS and the MAA. His interest was in getting mathematicians placed in universities or in wartime research; definitely the infantry was not the proper place. Later on during the Korean war he was to serve on the Selective Service Advisory Committee on Specialized Personnel, and with the same point of view.

His most concentrated period of service to the American Mathematical Society was no doubt the years 1950-1954. In the years 1950-1952 he was a member of the Transactions Editorial Board, and in 1951-1952 was Managing Editor of the Transactions. In 1952 he was President Elect of the Society and in 1953-1954 served as President. The years were interesting ones-the publication problems were mounting in their inexorable way, federal support for research was 
coming into being, the lingering political problems from the McCarthy era were working themselves out, the Society and the mathematical community were growing. After these years he continued to serve for several years as member of the Board of Trustees and to take a keen interest in the Society, but no doubt felt that he had finished his turn of concentrated work for the Society.

$\mathrm{He}$ also spent a fair amount of time in Washington in the fifties. In 1952 he was on the NSF Graduate Fellowships selection panel, and in 1953-1955 was chairman of that panel. Thereafter he served on the post-doctoral panel. In the years 1956-1959, he was on the Mathematical, Physical and Engineering Sciences Divisional Committee of the NSF. Throughout he kept up a lively interest in the development of the NSF. In an unusual way he was interested in the predominance of first-rate research, and at the same time for regional development.

After the busy years of the fifties, he relaxed his interest in the flow of national mathematics somewhat, but in a typical way would always be willing to put in a good word for principles he considered paramount, such as quality or basic research.

As was inevitable for one of such accomplishment in research as well as in administration and teaching, Whyburn received numerous awards and honors. In 1938 he received the Chauvenet Prize of the MAA for his expository paper [70], "On the structure of continua." In 1940, he was Colloquium Lecturer of the AMS; his book, Analytic lopology [84], grew out of those lectures. He was awarded the Sc.D. degree by Washington and Lee University in 1949. In 1951 he became a member of the National Academy of Sciences. In 1968 he received the Thomas Jefferson Award, the top award of the University of Virginia.

\section{HIS RESEARCH}

Although Whyburn's total body of mathematical research has a considerable unity about it, it is nevertheless desirable to divide it into a few categories. Needless to say, the following concerns only a sampling of his work.

1. Cyclic elements and the structure of continua. By a continuum we mean a compact connected metric space. Whyburn's early research involved understanding the full details about the connections between a plane continuum, of tentimes assumed to be locally connected, and the regions into which it divides the plane. In the course of this effort, he came upon a structure theory for locally connected plane continua. Within a very few years, this theory turned out to 
hold for arbitrary locally connected continua. The applications for plane continua turned out to be just one facet of the theory. We first indicate the final result, cyclic element theory [35], [84 ].

Let $X$ be a locally connected continuum. A point $x \in X$ is a cut point if $X-x$ fails to be connected; it is an end point if for each $\epsilon>0$ there exists an open set $U$ with $x \in U$ and diam $U<\epsilon$ such that $\partial U=\vec{U}-U$ consists of a single point. Define $X$ to be cyclic if any two points of $X$ are contained in a simple closed curve of $X$. In the cyclic connectedness theorem [8], [47], Whyburn proved that a locally connected continuum is cyclic if and only if it has no cut points. The basic idea of cyclic element theory can be put this way: one can understand a locally connected continuum completely if one can understand the locally connected cyclic subcontinua which are maximal with respect to being cyclic.

Define a true cyclic element of $X$ to be a connected subset, consisting of more than one point, which is maximal with respect to having no cut points of itself.

TheOREM. Let $X$ be a locally connected continuum, and let $x \in X$ be neither a cut point nor an end point. Then $x$ is contained in a unique true cyclic element. A true cyclic element is a locally connected cyclic continuum. $X$ has at most a countable number of true cyclic elements, and their diameters tend to zero. Any two of them intersect in at most a point, and the point of intersection must be a cut point.

Among the most basic of the papers of Whyburn which can be classified as contributing to cyclic element theory are [2], [4], [8], $[9],[14],[15],[16],[30],[31],[32],[35],[47],[48],[59],[80]$. And in addition to the broad scope of the theory, these papers are replete with lemmas and theorems which have found many uses elsewhere, e.g., the cut point order theorem.

An end point of a continuum $X$ is of order 1 in $X$. A non-end point $x \in X$ is of order 2 in $X$ if for each $\epsilon>0$ there exists an open set $U$ with $x \in U$ such that diam $U<\epsilon$ and $\partial U$ consists of exactly two points.

TheOREM. All except possibly a countable number of the cut points of a continuum $X$ are of order 2 in $X$.

Whyburn generalized this theorem in at least two directions, to noncompact connected spaces in particular.

At the same time as the basic cyclic element theory was developing, the applications were being made. Define a boundary curve to be a locally connected continuum each true cyclic element of which is a simple closed curve. If $X$ is a locally connected continuum in the 
2-sphere $S^{2}$ which is the boundary of a connected open set, then $X$ is a boundary curve [16]. Given a boundary curve $X$, there is a homeomorphic image $X^{\prime}$ of $X$ which is the boundary of a connected open set (this is due to Ayres). Moreover, if $X$ is a locally connected continuum in $S^{2}$ which does not separate $S^{2}$, then each true cyclic element is a two-cell [16]. Also, a locally connected continuum in $S^{2}$ fails to separate $S^{2}$ if and only if each true cyclic element fails to separate. This latter fact is the first property of a sort that was later called cyclicly extensible and reducible.

A cactoid is a locally connected continuum each true cyclic element of which is a 2-sphere. A map $f$ of a locally connected continuum onto a Hausdorff space $Y$ is monotone if each $f^{-1}(y)$ is a continuum. The following theorem is largely due to R. L. Moore, with a later addition by Whyburn.

THEOREM. Every monotone image of a cactoid is a cactoid. Every cactoid is the monotone image of a 2-sphere.

While on the subject, note that in 1934, Whyburn gave the proper class of maps which did for boundary curves what monotone maps did for cactoids [60]. Let $X$ and $Y$ be locally connected continua. A map $f: X \rightarrow Y$ is said to be nonalternating if whenever $y \in Y$ then any $f^{-1}\left(y^{\prime}\right)$ for $y^{\prime} \neq y$ is contained in a single component of $X-f^{-1}(y)$.

THEOREM. Every nonalternating image of a boundary curve is a boundary curve. Every boundary curve is the nonalternating image of a simple closed curve.

An excellent summary of the subject, including not only Whyburn's work but also the subsequent work, is the paper of B. L. McAllister, Amer. Math. Monthly 73 (1966), 337-350. Whyburn's book [84], Analytic topology, is the best source for a full treatment including proofs.

2. Regular convergence and monotone maps. For a period around 1933-1935, Whyburn became interested in homology theory and its possibilities for higher dimensional generalizations of structure theorems for continua and for maps. At least two of his contributions of this period have become of permanent interest. Recall that the space $2^{x}$ of closed subsets of a compact metric space has a natural topology, that of the Hausdorff metric. Convergence of closed subsets will mean convergence in this metric. The subset of $2^{x}$ consisting of all continua is closed in $2^{x}$. But, for example, if $X=S^{2}$ then sequences of arcs converge to some very weird limits indeed. Whyburn defined convergence in a much more restricted fashion so that the limit would be much nicer. 
A sequence $\left(A_{n}\right)$ of closed subsets of a compact metric space $X$ is said to converge regularly to the closed subset $A$ [64] if $\left(A_{n}\right)$ converges to $A$ and if given $\epsilon>0$ there exists $\delta>0$ and an integer $N$ such that if $n>N$ then any two points $x, y$ of $A_{n}$ with $\rho(x, y)<\delta$ are contained in a connected subset of $A_{n}$ of diameter $<\epsilon$. The definition for $r$-regular convergence is similar: for $n>N$, any Vietoris cycle (over the integers mod 2) of dimension $\leqq r$ of diameter $<\delta$ bounds in a subset of $A_{n}$ of diameter $<\epsilon$. Then 0 -regular convergence $=$ regular convergence. Moreover the sequence $A, A, \cdots, A, \cdots$ converges $r$-regularly to $A$ if and only if $A$ is lc $^{r}$ (that is, is locally connected in dimensions $\leqq r$ in the sense of homology over the integers mod 2).

In current language, the language can be easily put in terms of Cech cohomology over any coefficient group $K$. It is required that $\left(A_{n}\right)$ converge to $A$ and that given $x \in X$ and a closed neighborhood $U$ of $x$ in $X$ then there exist a closed neighborhood $V \subset U$ of $x$ and an $N$ such that for $n>N$,

$$
\widetilde{H}^{i}\left(U, \cap, A_{n} ; K\right) \rightarrow \widetilde{H}^{i}\left(V \cap A_{n} ; K\right)
$$

has trivial image for $i=0,1, \cdots, r$.

Whyburn's interest in the concept was for such theorems as the following [64].

Theorem. Let the sequence $A_{1}, A_{2}, \cdots$ of 2-spheres converge $i$-regularly to the closed subset $A$ of a compact metric space. If $i=0$, then $A$ is a cactoid. If $i=1$, then $A$ is either a 2-sphere or a single point.

A number of people have considered the concept over the years. For example, if $\left(A_{n}\right)$ converges $r$-regularly to $A$, then

$$
H^{i}\left(A_{n} ; K\right) \approx H^{i}(A ; K), \quad i=0,1, \cdots, r,
$$

for $n$ large. Moreover $A$ is $\mathrm{lc}^{r}$. For a survey of some years ago, see Paul A. White, Bull. Amer. Math. Soc. 60 (1954), 431-443.

Whyburn had also begun to encounter special instances of the following phenomenon. Given compact metric spaces $X$ and $Y$, there is the space $Y^{X}$ of continuous maps of $X$ into $Y$. There are also natural subsets $\mathfrak{M} \subset Y^{\boldsymbol{X}}$ (for example, all monotone maps). In some instances, the subset of elements of $\mathscr{T}$ which map $X$ onto $Y$ is closed. More generally, if one puts restrictions on the fashion in which the images converge, the limit of a convergent sequence of elements of 9 may be in $\mathfrak{T}$.

He defined $f: X \rightarrow Y$ to be $r$-monotone if each $f^{-1}(y)$ is acyclic in dimensions $\leqq r$ (that is, has vanishing reduced Cech homology groups over the integers mod 2 in dimensions $\leqq r$ ). Thus 0 -monotone $=$ monotone. 
Theorem [65]. Let $X$ and $W$ be compact metric spaces and let $\left(f_{n}\right)$ be a sequence of $r$-monotone maps of $X$ into $W$; let $Y_{n}=f_{n}(X)$. Suppose the sequence $\left(f_{n}\right)$ converges uniformly to the map $f$ of $X$ onto $Y \subset W$. Then $\left(Y_{n}\right)$ converges $r$-regularly to $Y$ if and only if $Y$ is $\mathrm{lc}^{r}$ and $f$ is $r$-monotone.

Note the corollary. If each $f_{n}$ is an $r$-monotone map of $X$ onto the lcr space $Y$ and if $\left(f_{n}\right)$ converges uniformly to $f$, then $f$ is $r$-monotone. Let $r=0$. If each $f_{n}$ is a monotone map of $X$ onto the locally connected compact metric space $Y$, and if $\left(f_{n}\right)$ converges uniformly to $f$, then $f$ is monotone.

As a corollary, if $\left(f_{n}\right)$ is a sequence of homeomorphisms of $S^{2}$ onto $S^{2}$ which converges uniformly to $f$, then $f$ is monotone. J. W. T. Youngs later proved the converse: every monotone map of $S^{2}$ onto $S^{2}$ is a uniform limit of homeomorphisms.

In later years, Whyburn was considering noncompact domains and ranges, and considered questions for the noncompact case similar to the above. See [114], [115], [129].

3. Open maps. Around 1936, Whyburn began to consider the work of Stoilow. Call a map $f: X \rightarrow Y$ open if whenever $U$ is open in $X$ then $f(U)$ is open in $Y$. Call $f$ light if each $f^{-1}(y)$ is totally disconnected.

TheOREM (Stollow). Let $X$ be an orientable 2-manifold without boundary and let $f: X \rightarrow S^{2}$ be a map. Then $f$ is light and open if and only if there exist a Riemann surface $R$, a homeomorphism $h$ of $X$ onto $R$, and a nonconstant complex analytic map $g: R \rightarrow S^{2}$ such that $f=g h$.

By now Whyburn had encountered a number of instances (see \$1) of the following: a narrow class $\mathcal{S}$ of spaces and a wide class $\mathfrak{T}$ of maps such that every $\mathfrak{T}$-image of an element of $S$ is also an element of $S$.

He considered now $S=2$-manifolds and $\mathfrak{T}=$ light open maps and proved the following [76].

Theorem. Let $X$ be a 2-manifold (with or without boundary) and let $f$ be a light open map of $X$ onto a Hausdorff space $Y$. Then $Y$ is a 2-manifold.

Moreover each $f^{-1}(y)$ is a discrete set. If $x \in f^{-1}(y)$ is not a boundary point of $X$, there exist coordinate neighborhoods $U$ of $x$ and $V$ of $y$ such that $f(U)=V$ and

(i) $f: U \rightarrow V$ is equivalent to the $\operatorname{map} f^{\prime}(z)=z^{p}$ of $\{z:|z|<1\}$ onto itself (in case $y$ is not on the boundary of $Y$ ), or

(ii) $f: U \rightarrow V$ is equivalent to $f^{\prime \prime} f^{\prime}$, where $f^{\prime \prime}(x+i y)=x+i|y|$, in case $y$ is on the boundary.

If $X$ is compact, then $f$ is simplicial in appropriate subdivisions of 
$X$ and $Y$. If $Y$ is a closed orientable surface so also is $X$. His papers [74], [76], [77], [88], [102], [125] treat open maps on 2-manifolds and related questions.

Off and on for the rest of his life, Whyburn was to spend considerable time on open maps, particularly on lines of research relating their topological properties to complex function theory. For example, consider the classical theorem that if a sequence $\left\{f_{n}\right\}$ of complex analytic functions converges uniformly on compact sets to a limit $f$, then $f$ is complex analytic. However on the purely topological side the sets $\Re \subset Y^{\boldsymbol{X}}$ of open maps, or light open maps, are not closed. For as we have seen, any monotone map of $S^{2}$ onto $S^{2}$ is the uniform limit of a sequence of homeomorphisms. He arrived at the notion "pseudo-open" as the correct limit concept [89], [97], [101], [112]. A map $f$ of a locally connected, locally compact separable metric space $X$ into another $Y$ is pseudo-open if whenever $U$ is an open set containing a compact component of some $f^{-1}(y)$, then $f(U)$ contains $y$ in its interior.

THEOREM [112]. Suppose $\left(f_{n}\right)$ is a sequence of pseudo-open maps of $X$ onto $Y$ ( $X$ and $Y$ as above) which converges uniformly on compact sets to $f: X \rightarrow Y$. Then $f$ is pseudo-open. In particular if $f$ is light then $f$ is also open.

As Whyburn thought about the light open maps on 2-manifolds as the topological counterparts of the complex analytic functions, it annoyed him that analytic machinery was needed to prove the nonconstant complex analytic functions light and open. Ursell and Eggleston provided a proof using essentially no analytic machinery. It then seemed to Whyburn that one should go ahead and prove topologically, assuming only the existence of the derivative, that they had a second derivative, etc. He publicized the problem, in the first edition of his book, Topological analysis [117] and elsewhere [106]. Plunkett made considerable progress and in a few years Connell and Connell-Porcelli wiped out the problem completely. For a complete account, see the revised edition of his book [117] and see also [128]. In fact, the revised edition of his book may be used as a starting place for reading his work on open maps.

4. Compact maps and quotient maps. In considering light open maps $f$ from one 2-manifold on to another, even if the map is finite-toone there is not necessarily a finite degree for the map. In examining such problems, Whyburn was led to the compact maps [101], maps $f: X \rightarrow Y$ such that whenever $K$ is compact in $Y$ then $f^{-1}(K)$ is com- 
pact in $X$. It turned out that Vainstein had already considered such maps in 1947, and had proved that closed maps with compact point inverses were compact. Whyburn was later to prove [133] that if $Y$ is a $k$-space then compact maps are also closed.

In 1953, he proved [105], [136] that every map is the restriction of a compact map. Consider an arbitrary map $f: X \rightarrow Y$ where $X$ and $Y$ are $T_{1}$-spaces. Denote by $Z=X+Y$ the disjoint union of $X$ and $Y$. Define $Q \subset Z$ to be open if

(i) $Q \cap X$ and $Q \cap Y$ are open,

(ii) for any compact $K \subset Q \cap Y, f^{-1}(K) \cap(X-Q)$ is compact.

One can regard $X$ as open in $Z$ and $Y$ as closed in $Z$. A retraction $r$ of $Z$ onto $Y$ is defined by $r=f$ on $X, r=$ id on $Y$. The restriction $r \mid X$ is $f$, and $r$ is a compact map.

Whyburn was interested in maps $f: X \rightarrow Y$ with each $f^{-1}(y)$ compact. When is $f$ a compact map? He proved [119] that every map $f$ of the line into the line with compact point inverses is compact. However with $X=Y=$ plane, this is false but one has instead [119]:

THEOREM. Every monotone map of the plane onto the plane is compact.

He raised the question of generalizations to higher dimensions [127]. Among a number of interesting responses, Vaisala proved that every $(n-2)$-monotone map of $E^{n}$ onto $E^{n}$ is compact. In the other direction, Bing in 1969 proved the existence of monotone maps of $E^{3}$ on to $E^{3}$ which are not compact.

Whyburn was also interested in quotient maps (which he called quasi-compact maps). He observed that if $f$ is a quotient map of $X$ onto $Y$ then it was not necessarily true that $f: f^{-1}(A) \rightarrow A$ was a quotient map for any $A \subset Y$. In fact, he proved that $f: f^{-1}(A) \rightarrow A$ is a quotient map for all $A \subset Y$ (and all $f$ ) if and only if $Y$ is an accessibility space; that is, given $A$ in $Y$ with limit point $p$ there is $B C A \cup p$ with $B$ closed and with $p$ a limit point of $B$ [109], [146]. For a first place to read about such theorems, see his last paper [149].

\section{His teaching}

Some teachers are admired for their brilliant and inspiring lectures, others for their precise conciseness, and still others for their ability to dramatize the subject. Whyburn was none of these. His natural modesty precluded showiness, and he felt that understanding was more an internal than an external matter. He liked to give the students something they could do. In this way, not only was understanding achieved, but confidence and ability were strengthened as well.

He did inspire his students, not in an instant, but more slowly and 
over a longer period of time. Here again continuity was a principlecontinuity of effort-continuity of interest in the individual student. He knew that confidence came slowly, for he had himself wondered as he committed himself to mathematics, if he would be able to think of new things to do. However it was, whether by example and personal devotion to research, or by understanding or encouragement, clear that he inspired and attracted students. Many were influenced by him and most of his own doctoral students developed successful research careers of their own.

Ph.D. students of G. T. Whyburn. At The Johns Hopkins University: C. H. Harry (1932), Barbara Aitchison (1933), C. H. Wheeler (1933), G. E. Schweigart (1934), J. F. Wardwell (1935). At the University of Virginia: D. W. Hall (1938), A. D. Wallace (1939), J. L. Kelley (1940), P. A. White (1942), C. L. Clark (1944), E. E. Floyd (1948), M. K. Fort (1948), R. H. Kasriel (1953), R. L. Plunkett (1953), R. F. Williams (1954), W. E. Malbon (1955), R. W. Jollensten (1956), P. E. McDougle (1958), Edwin Duda (1961), G. K. Williams (1964), R. A. Duke (1965), R. F. Dickman (1966), E. A. Stone (1966), A. C. Garcia Maynez (1968), Evelyn McMillan (1968).

\section{Bibliography of G. T. WhyburN}

1. Concerning certain types of continuous curves, Proc. Nat. Acad. Sci. U.S.A. 12 (1926), 761-767.

2. Two-way continuous curves, Bull. Amer. Math. Soc. 32 (1926), 659-662.

3. Concerning connected and regular point sets, Bull. Amer. Math. Soc. 33 (1927), 685-689.

4. Concerning continua in the plane, Trans. Amer. Math. Soc. 29 (1927), 369-400.

5. Concerning point sets which can be made connected by the addition of a simple continuous arc, Trans. Amer. Math. Soc. 29 (1927), 746-754.

6. Concerning the disconnection of continua by the omission of pairs of their points, Fund. Math. 10 (1927), 180-185.

7. Concerning the open subsets of a plane continuous curve, Proc. Nat. Acad. Sci. U.S.A. 13 (1927), 650-657. 31-38.

8. Cyclicly connected continuous curves, Proc. Nat. Acad. Sci. U.S.A. 13 (1927),

9. Some properties of continuous curves, Bull. Amer. Math. Soc. 33 (1927), 305-308.

10. The most general closed point set over which a continuous function may be defined by certain properties, Bull. Amer. Math. Soc. 33 (1927), 185-188.

11. Concerning accessibility in the plane and regular accessibility in $n$ dimensions, Bull. Amer. Math. Soc. 34 (1928), 504-510.

12. Concerning Menger regular curves, Fund. Math. 12 (1928), 264-294.

13. Concerning plane closed point sets which are accessible from certain subsets of their complements, Proc. Nat. Acad. Sci. U.S.A. 14 (1928), 657-666.

14. Concerning the complementary domains of continua, Ann. of Math. 29 (1928), 399-411.

15. Concerning the cut points of continua, Trans. Amer. Math. Soc. 30 (1928), 597-609. 
16. Concerning the structure of a continuous curve, Amer. J. Math. 50 (1928), 167194.

17. On a problem of W. L. Ayres, Fund. Math. 11 (1928), 298-301.

18. On certain accessible points of plane continua, Monatsh. Math. Phys. 35 (1928), 289-304.

19. (with W. L. Ayres), On continuous curves in n-dimensions, Bull. Amer. Math. Soc. 34 (1928), 349-360.

20. (with J. R. Bailey), The behavior of 2-phenyl semicarbazones upon oxidation, J. Amer. Chem. Soc. 50 (1928), 905-912.

21. A generalized notion of accessibility, Fund. Math. 14 (1929), 311-326.

22. Concerning collections of cuttings of connected point sets, Bull. Amer. Math. Soc. 35 (1929), 87-104.

23. Concerning points of continuous curves defined by certain im kleinem properties, Math. Ann. 102 (1929), 313-336.

24. Continuous curves and arc-sums, Fund. Math. 14 (1929), 103-106.

25. Concerning irreducible cuttings of continua, Fund. Math. 13 (1929), 42-57.

26. Local separating points of continua, Monatsh. Math. Phys. 36 (1929), 305-314.

27. On regular points of continua and regular curves of at most order $n$, Bull. Amer. Math. Soc, 35 (1929), 218-224.

28. On simple closed curves, Bull. Internat. Acad. Polon. Sci. Lett. Cl. Sci. Math.Nat. Ser. A. 1929, no. 6A, 280-293.

29. A continuum every subcontinuum of which separates the plane, Amer. J. Math. 52 (1930), 319-330.

30. Cut points of connected sets and of continua, Trans. Amer. Math. Soc. 32 (1930), 147-154. 194.

31. On the set of all cut points of a continuous curve, Fund. Math. 15 (1930), 185-

32. On the structure of connected and connected im kleinem point sets, Trans. Amer. Math. Soc. 32 (1930), 926-943.

33. Potentially regular point sets, Fund. Math. 16 (1930), 160-172.

34. Sur l'accessibilité des continus plans, Fund. Math. 15 (1930), 322-323.

35. (with C. Kuratowski), Sur les êlèments cycliques et leurs applications, Fund. Math. 16 (1930), 305-331.

36. Sur les e-séparations irréductibles, Fund. Math. 16 (1930), 77-80.

37. The rationality of certain continuous curves, Bull. Amer. Math. Soc. 36 (1930), 522-524.

38. A junction property of locally connected sets, Amer. J. Math. 53 (1931), 753-756.

39. Concerning addition of regular curves, Monatsh. Math. Phys. 38 (1931), 1-4.

40. Concerning continuous images of the interval, Amer. J. Math. 53 (1931), 670674.

41. Concerning hereditarily locally connected continua, Amer. J. Math. 53 (1931), 374-384.

42. Concerning the proposition that every closed compact and totally disconnected set of points is a subset of an arc, Fund. Math. 18 (1931), 47-60.

43. Concerning the subsets of regular curves, Monatsh. Math. Phys. 38 (1931), $85-88$.

44. Continuous curves without local separating points, Amer. J. Math. 53 (1931), 163-166.

45. Non-separated cuttings of connected point sets, Trans. Amer. Math. Soc. 33 (1931), 444-454. 
46. On the divisiblity of locally connected spaces, Bull. Amer. Math. Soc. 37 (1931), 734-736.

47. On the cyclic connectivity theorem, Bull. Amer. Math. Soc. 37 (1931), 429-433.

48. The cyclic and higher connectivity of locally connected spaces, Amer. J. Math. 53 (1931), 427-442.

49. A certain transformation on metric spaces, Amer. J. Math. 54 (1932), 367-375.

50. A note on spaces which have the $S$ property, Amer. J. Math. 54 (1932), 536-538.

51. On the decomposability of closed sets into a countable number of simple sets of various types, Amer. J. Math. 54 (1932), 169-175.

52. On the construction of simple arcs, Amer. J. Math. 54 (1932), 518-524.

53. Characterization of certain curves by continuous functions defined upon them, Amer. J. Math. 55 (1933), 131-134.

54. Concerning S-regions in locally connected continua, Fund. Math. 20 (1933), 131-139.

55. Decompositions of continua by means of local separating points, Amer. J. Math. 55 (1933), 437-457.

56. On the existence of totally imperfect and punctiform connected subsets in a given continuum, Amer. J. Math. 55 (1933), 146-152.

57. Sets of local separating points of a continuum, Bull. Amer. Math. Soc. 39 (1933), $97-100$

58. Concerning maximal sets, Bull. Amer. Math. Soc. 40 (1934), 159-164.

59. Cyclic elements of higher orders, Amer. J. Math. 56 (1934), 133-146.

60. Non-alternating transformations, Amer. J. Math. 56 (1934), 294-302. 96.

61. A decomposition theorem for closed sets, Bull. Amer. Math. Soc, 41 (1935), 95-

62. Concerning continua of finite degree and local separating points, Amer. J. Math. 57 (1935), 11-16.

63. Generalized perfect sets, Duke Math. J. 1 (1935), 35-38.

64. On sequences and limiting sets, Fund. Math. 25 (1935), 408-426.

65. Regular convergence and monotone transformations, Amer. J. Math. 57 (1935), 902-906.

66. Arc preserving transformations, Amer. J. Math. 58 (1936), 305-312.

67. Completely alternating transformations, Fund. Math. 27 (1936), 140-146.

68. Concerning rationality bases for curves, Ergebnisse eines, Math. Kolloq. 7 (1936), 58-60.

69. On continua of condensation, Amer. J. Math. 58 (1936), 705-708.

70. On the structure of continua, Bull. Amer. Math. Soc. 42 (1936), 49-73.

71. Semi-closed sets and collections, Duke Math. J. 2 (1936), 685-690.

72. Interior transformations on compact sets, Duke Math. J. 3 (1937), 370-381.

73. A theorem on interior transformations, Bull. Amer. Math. Soc. 44 (1938), 414416.

74. Interior surface transformations, Duke Math. J. 4 (1938), 626-634.

75. Interior transformations on certain curves, Duke Math. J. 4 (1938), 607-612.

76. Interior transformations on surfaces, Amer. J. Math. 60 (1938), 477-490.

77. The mapping of Betti groups under interior transformations, Duke Math. J. 4 (1938), 1-8.

78. Non-alternating interior retracting transformations, Ann, of Math. 40 (1939), 914-921. MR 1, 45.

79. On irreducibility of transformations, Amer. J. Math. 61 (1939), 820-822. MR 1,45 . 
80. Semi-locally connected sets, Amer. J. Math. 61 (1939), 733-749. MR 1, 31.

81. The existence of certain transformations, Duke Math. J. 5 (1939), 647-655. MR 1,30 .

82. A relation between non-alternating and interior transformations, Bull. Amer. Math. Soc. 46 (1940), 320-321. MR 1, 319.

83. (with D. W. Hall), Arc- and tree-preserving transformations, Trans. Amer. Math. Soc. 48 (1940), 63-71. MR 1, 319.

84. Analytic topology, Amer. Math. Soc. Colloq. Publ., vol. 28, Amer. Math. Soc., Providence, R.I., 1942; rev. ed., 1955. MR 4, 86.

85. On the interiority of real functions, Bull. Amer. Math. Soc. 48 (1942), 942-945. MR 4, 224.

86. What is a curve? Amer. Math. Monthly 49 (1942), 493-497; Studies in Modern Topology, Prentice-Hall, Englewood Cliffs, N. J., 1968, pp. 23-38. MR 4, 89; MR 37 \#895.

87. Homotopy reductions of mappings into the circle, Duke Math. J. 11 (1944), 3542. MR 5, 213.

88. Interior mappings into the circle, Duke Math. J. 11 (1944), 431-434. MR 6, 164.

89. Topological analog of the Weierstrass double series theorem, Bull. Amer. Math. Soc. 50 (1944), 242-245. MR 5, 274.

90. Boundary alternation of monotone mappings, Duke Math. J. 12 (1945), 663667. MR 7, 336.

91. Coherent and saturated collections, Trans. Amer. Math. Soc. 57 (1945), 287-298. MR 6, 182.

92. Extensions of plane continua mappings, Amer. J. Math. 67 (1945), 505-520. MR 7, 136.

93. Uniqueness of the inverse of a transformation, Duke Math. J. 12 (1945), 317323. MR 7, 36.

94. On monotone retractability into simple arcs, Bull. Amer. Math. Soc. 52 (1946), 109-112. MR 7, 468. 196.

95. On locally simple curves, Bull. Amer. Math. Soc. 53 (1947), 986-992. MR 9,

96. On n-arc connectedness, Trans. Amer. Math. Soc. 63 (1948), 452-456. MR 10, 138.

97. Sequence approximations to interior mappings, Ann. Soc. Polon. Math. 21 (1948), 147-152. MR 10, 261.

98. Continuous decompositions, Amer. J. Math. 71 (1949), 218-226. MR 10, 317.

99. Modern development of mathematics, Va. J. Science 1 (1950), 93-102.

100. Open and closed mappings, Duke Math. J. 17 (1950), 69-74. MR 11, 194.

101. Open mappings on locally compact spaces, Mem. Amer. Math. Soc. No. 1, (1950), 24 pp. MR 13, 764.

102. An open mapping approach to Hurwitz's theorem, Trans. Amer. Math. Soc. 71 (1951), 113-119. MR 13, 149.

103. On k-fold irreducibility of mappings, Amer. J. Math. 74 (1952), 910-912. MR 14, 305.

104. On quasi-compact mappings, Duke Math. J. 19 (1952), 445-446. MR 14, 192.

105. A unified space for mappings, Trans. Amer. Math. Soc. 74 (1953), 344-350. MR 14, 669.

106. Introductory topological analysis, Lectures on Functions of a Complex Variable, Univ. of Michigan Press, Ann Arbor, Mich., 1955, pp. 1-14. MR 16, 1140. 
107. Relative quasi-compactness of mappings, Proc. Nat. Acad. Sci. U.S.A. 41 (1955), 974-978. MR 19, 568.

108. Set-theoretic topology-present and future, and Structure of continua, Summary of Lectures and Seminars, Summer Institute on Set Theoretic Topology, 1955, pp. 6, 67-68.

109. Mappings on inverse sets, Duke Math. J. 23 (1956), 237-240. MR 20 \#4822.

110. Topological analysis, Bull. Amer. Math. Soc. 62 (1956), 204-218. MR 17, 1229.

111. Dimension and non-density preservation of mappings, Pacific J. Math. 7 (1957), 1243-1249. MR 20 \#1299.

112. Quasi-open mappings, Rev. Math. Pures Appl. 2 (1957), 47-52. MR 20 \#1300.

113. Sense and orientation on the disk, Amer. Math. Monthly 64 (1957), no. 8, part II, 103-106. MR 20 \#4816.

114. Uniform convergence for monotone mappings, Proc. Nat. Acad. Sci. U.S.A. 43 (1957), 992-998. MR 20 \#280.

115. On convergence of mappings, Colloq. Math. 6 (1958), 311-318. MR 20 \#256.

116. On the invariance of openness, Proc. Nat. Acad. Sci. U.S.A. 44 (1958), 464466. MR 21 \#2216.

117. Topological analysis, Princeton Math. Series, no. 23, Princeton Univ. Press, Princeton, N.J., 1958; 2nd rev. ed., 1964. MR 20 \#6081; MR 29 \#2758.

118. Topological characterization of the Sierpinski curve, Fund. Math. 45 (1958), 320-324. MR 20 \#6077.

119. Compactness of certain mappings, Amer. J. Math. 81 (1959), 306-314. MR 22 \#1881.

120. Mapping norms, Proc. Nat. Acad. Sci. U.S.A. 45 (1959), 1431-1436. MR 21 \#6578.

121. Convergence in norm, Proc. Nat. Acad. Sci. U.S.A. 46 (1960), 1614-1617. MR 22 \#8476.

122. Non-obstructing sets and related mappings, Proc. Nat. Acad. Sci. U.S.A. 46 (1960), 1244-1247. MR 23 \#A624.

123. Norms for open mappings, J. London Math. Soc. 35 (1960), 302-309. MR 23 \#1825.

124. Compressibility and uniform convergence, Proc. Nat. Acad. Sci. U.S.A. 47 (1961), 1843-1847. MR 24 \#A2372.

125. Open mappings on 2-dimensional manifolds, J. Math. Mech. 10 (1961), 181197. MR 27 \#2967.

126. A measure distortion mapping, Proc. Nat. Acad. Sci. U.S.A. 48 (1962), 19221924. MR $26 \# 1857$.

127. Decomposition spaces, Topology of 3-manifolds and Related Topics (Proc. Univ. of Georgia Inst., 1961) Prentice-Hall, Englewood Cliffs, N.J., 1962, pp. 2-4. MR 25 \#4499.

128. Developments in topological analysis, Fund. Math. 50 (1961/62), 305-318. MR $29 \# 3651$.

129. Monotoneity of limit mappings, Duke Math. J. 29 (1962), 465-470. MR 26 \#6935.

130. The Cauchy inequality in topological analysis, Proc. Nat. Acad. Sci. U.S.A. 48 (1962), 1335-1336. MR 32 \#281.

131. Generic and related mappings, Bull. Amer. Math. Soc. 69 (1963), 757-761. MR 28 \#1588. 
132. Simplification of mappings, Proc. Nat. Acad. Sci. U.S.A. 50 (1963), 431-435. MR 27 \#4210.

133. On compactness of mappings, Proc. Nat. Acad. Sci. U.S.A. 52 (1964), 14261431. MR $31 \# 722$.

134. Continuity of multifunctions, Proc. Nat. Acad. Sci. U.S.A. 54 (1965), 14941501. MR $32 \# 6423$.

135. Directed families of sets and closedness of functions, Proc. Nat. Acad. Sci. U.S.A. 54 (1965), 688-692. MR 32 \#435.

136. Compactification of mappings, Math. Ann. 166 (1966), 168-174. MR 34 \#791.

137. Connectivity of peripherally continuous functions, Proc. Nat. Acad. Sci. U.S.A. 55 (1966), 1040-1041. MR 33 \#4902.

138. Functions and multifunctions (assisted by John H. V. Hunt), University of Virginia Lecture Notes, 1966/67.

139. General topology and mapping theory (assisted by R. F. Dickman, 1964/65; revised in 1966/67 with assistance of John H. V. Hunt, A. C. Garcia-Maynez and Richard Sarchet), University of Virginia Lecture Notes.

140. Loosely closed sets and partially continuous functions, Michigan Math. J. 14 (1967), 193-205. MR 34 \#8387.

141. Quasi-closed sets and fixed points, Proc. Nat. Acad. Sci. U.S.A. 57 (1967), 201-205. MR 35 \#1006.

142. Cut points in general topological spaces, Proc. Nat. Acad. Sci. U.S.A. 61 (1968), 380-387. MR 39 \#3463.

143. Introductory topology (with the assistance of W. C. Chewning and Margaret Moody), University of Virginia Lecture Notes (manuscript).

144. Retracting multifunctions, Proc. Nat. Acad. Sci. U.S.A. 59 (1968), 343-348. MR 37 \#3543.

145. Inward motions in connected spaces, Proc. Nat. Acad. Sci. U.S.A. 63 (1969), 271-274.

146. Accessibility spaces, Proc. Amer. Math. Soc. 24 (1970), 181-185. MR 40\# 1973.

147. Locally cohesive spaces, Proc. Kanpur Topological Conference, 1968.

148. Functional movements in dendritic structures, Proc. Kanpur Topological Conference, 1968.

149. Dynamic topology, Amer. Math. Monthly 77 (1970), 556-570.

University of Virginia, Charlottesville, Virginia 22903

University of California, Riverside, California 92507 